\title{
Convexification of Power Flow Problem over Arbitrary Networks
}

\author{
Ramtin Madani, Javad Lavaei and Ross Baldick
}

\begin{abstract}
Consider an arbitrary power network with PV and PQ buses, where active powers and voltage magnitudes are known at PV buses, and active and reactive powers are known at PQ buses. The classical power flow (PF) problem aims to find the unknown complex voltages at all buses. This problem is usually solved approximately through linearization or in an asymptotic sense using Newton's method, given that the solution belongs to a good regime containing voltage vectors with small angles. The question arises as to whether the PF problem can be cast as the solution of a convex optimization problem over that regime. The objective of this paper is to show that the answer to the above question is affirmative. More precisely, we propose a class of convex optimization problems with the property that they all solve the PF problem as long as angles are small. Each convex problem proposed in this work is in the form of a semidefinite program (SDP). Associated with each SDP, we explicitly characterize the set of complex voltages that can be recovered via that convex problem. Since there are infinitely many SDP problems, each capable of recovering a potentially different set of voltages, designing a good SDP problem is cast as a convex problem.
\end{abstract}

\section{INTRODUCTION}

The flows in an electrical grid are described by nonlinear AC power flow equations. This paper is concerned with the problem of finding an unknown vector of complex voltages $\mathbf{V} \in \mathbb{C}^{n}$ for an $n$-bus power system to satisfy $m$ quadratic constraints associated with $m$ known quantities measured or specified in the network. This general power flow problem can be formulated as

$$
\begin{array}{ll}
\text { find } & \mathbf{V} \in \mathbb{C}^{n} \\
\text { subject to } & \left\langle\mathbf{V} \mathbf{V}^{*}, \mathbf{M}_{i}\right\rangle=X_{i}, \quad i=1, \ldots, m
\end{array}
$$

where $\langle\cdot, \cdot\rangle$ represents the Frobenius inner product of matrices, $\mathbf{M}_{1}, \ldots, \mathbf{M}_{m}$ 's are certain $n \times n$ Hermitian matrices obtained from the admittance matrix of the power network, and $X_{1}, \ldots, X_{m}$ represent specified physical quantities such as the net active power, reactive power or squared voltage magnitude at a bus or the flow over a line of the network. Checking the existence of a solution to the quadratic feasibility problem (1) is NP-hard for both transmission and distribution networks due to their reduction to the subset sum problem [1], [2].

Since problem (1) is central to the analysis and operation of power systems, its high computational complexity motivates obtaining a tractable formulation of the power flow equations. Addressing this problem facilitates performing several key

Ramtin Madani and Javad Lavaei are with the Electrical Engineering Department, Columbia University (madani@ee.columbia.edu and lavaei@ee.columbia. edu). Ross Baldick is with the Department of Electrical and Computer Engineering, University of Texas at Austin (baldick@ece.utexas.edu). This work was supported by the NSF CAREER Award 1351279 and NSF EECS Award 1406865. tasks related to economic dispatch and state estimation for power networks.

\section{A. Semidefinite Programming Relaxation}

To tackle the non-convexity of the feasible set of the power flow problem, the semidefinite programming (SDP) relaxation technique could be deployed. By defining $\mathbf{W} \triangleq \mathbf{V V}^{*}$, the quadratic equations in (1) can be linearly formulated in terms of W. Motivated by this exact linearization, consider the matrix feasibility problem

$$
\begin{array}{ll}
\text { find } & \mathbf{W} \in \mathbb{H}_{+}^{n} \\
\text { subject to } & \left\langle\mathbf{W}, \mathbf{M}_{i}\right\rangle=X_{i}, \quad i=1, \ldots, m,
\end{array}
$$

where $\mathbb{H}_{+}^{n}$ is the set of $n \times n$ Hermitian positive semidefinite matrices. Solving the non-convex power flow problem (1) is tantamount to finding a rank-1 solution $\mathbf{W}$ for the above linear matrix inequality (because $\mathbf{W}$ could then be decomposed as $\mathbf{V V}^{*}$ ). The problem (2) is regarded as a convex relaxation of (1) since it includes no restriction on the rank of $\mathbf{W}$. Although (1) normally has a finite number of solutions whenever $m \geq 2 n-1$, its SDP relaxation (2) is expected to have infinitely many solutions because the matrix variable $\mathbf{W}$ includes $O\left(n^{2}\right)$ scalar variables as opposed to $O(n)$. However, under some additional assumptions, it is known in some applications, such as phase retrieval, that the relaxed problem would have a unique solution if $m \geq 3 n$, and that the solution has automatically rank-1 [3]. In the case where the relaxed problem does not have a unique solution, the literature of compressed sensing substantiates that minimizing trace $\{\mathbf{W}\}$ over the feasible set of (2) may yield a rank-1 matrix $\mathbf{W}$ under strong technical assumptions [4], [3], [5]. The main purpose of this paper is to study what objective function should be minimized (instead of trace $\{\mathbf{W}\}$ ) to attain a rank-1 solution for the power problem (2).

The potential of SDP relaxation for finding a global solution of the optimal power flow problem has been manifested in [6], with further studies conducted in [7], [8]. In addition, recent advances in leveraging the sparsity of network graph have made SDP problems computationally more tractable [9], [10], [11], [12], [13], [14]. In the case where the SDP relaxation fails to work, we have developed a graph-theoretic penalized SDP problem in [15] and [14], which attempts to identify the problematic lines of the network (sources of non-convexity) through a graph analysis and then penalize the loss over those lines in order to find a near-global solution for the optimal power flow problem. The proposed approach was successful in finding solutions with global optimality guarantees of at least $99 \%$ for IEEE and Polish test systems. 


\section{B. Classical Power Flow Problem}

Let $\mathcal{N}$ denote the set of buses of the power network under study, and let $P_{k}$ and $Q_{k}$ represent the net active and reactive power injections at every bus $k \in \mathcal{N}$. The complex voltage phasor at bus $k$ is denoted by $V_{k}$, whose magnitude and phase are shown as $\left|V_{k}\right|$ and $\measuredangle V_{k}$, respectively. One special case of (1) is the classical power flow (PF) problem, for which the number of quadratic constraints (namely $m$ ) is equal to $2 n-1$. To formulate the problem, three basic types of buses are considered, depending on the parameters that are known at each bus:

- PQ bus: $P_{k}$ and $Q_{k}$ are specified.

- PV bus: $P_{k}$ and $\left|V_{k}\right|$ are specified.

- The slack bus: $\left|V_{k}\right|$ is specified and $\measuredangle V_{k}$ is set to zero to serve as the reference for voltage angles.

Each PQ bus represents a load bus or possibly a generator bus, whereas each PV bus represents a generator bus. Given the specified parameters at every bus of the network, the classical $\mathrm{PF}$ problem aims to solve the network equations in order to find an operating point that fits the input values. This problem has been studied extensively for years, without much success in designing an advanced computational method that is able to outperform Newton's method in polar coordinates [16], [17], [18].

\section{Contributions}

This paper is aligned with the recent body of work on investigating the remarkable promises of SDP relaxations for power optimization problems. The major strength of Newton's method and similar traditional techniques is their local convergence property, meaning that the recovery of a feasible solution is possible as long as the starting point is sufficiently close to a solution. The main objective of this paper is to develop a similar property for the SDP relaxation. We propose a family of convex optimization problems of the form

$$
\begin{array}{ll}
\underset{\mathbf{W} \in \mathbb{H}^{n}}{\operatorname{minimize}} & \langle\mathbf{W}, \mathbf{M}\rangle \\
\text { subject to } & \left\langle\mathbf{W}, \mathbf{M}_{i}\right\rangle=X_{i}, \quad i=1, \ldots, m, \\
& \mathbf{W} \succeq 0,
\end{array}
$$

where the matrix $\mathbf{M} \in \mathbb{H}_{+}^{n}$ is to be designed. Unlike the compressing sensing literature that assumes $M=I$, we aim for systematically designing $\mathbf{M}$ such that the above problem yields a unique rank-1 solution $\mathbf{W}$ from which a feasible solution for the power flow problem (1) can be recovered. Notice that the existence of such a rank-1 solution depends in part on its input specifications $\left(X_{1}, X_{2}, \ldots, X_{m}\right)$.

Definition 1. It is said that the SDP problem (3) solves the nonlinear power flow problem (1) for the input $\left(X_{1}, X_{2}, \ldots, X_{m}\right)$ if (3) has a unique rank-1 solution. Given $\mathbf{M} \in \mathbb{H}^{n}$, define $\mathcal{M}_{X}(\mathbf{M}) \subseteq \mathbb{R}^{m}$ as the set of all specification vectors $\left(X_{1}, \ldots, X_{m}\right)$ for which the SDP problem (3) solves the nonlinear power flow problem (1).

Definition 2. Given $\mathbf{M} \in \mathbb{H}^{n}$, a voltage vector $\mathbf{V}$ is said be recoverable if $\mathbf{W}=\mathbf{V V}^{*}$ is a solution of the SDP problem
(3) for some $\left(X_{1}, \ldots, X_{m}\right)$ in $\mathcal{M}_{X}(\mathbf{M})$. Define $\mathcal{M}_{V}(\mathbf{M})$ as the set of all recoverable vectors $\mathbf{V}$.

Note that the SDP problem (3) can be used to find a solution of the nonlinear power flow problem (1) if and only if the provided specification set $\left(X_{1}, X_{2}, \ldots, X_{m}\right)$ belongs to $\mathcal{M}_{X}(\mathbf{M})$. In addition, the set $\mathcal{M}_{V}(\mathbf{M})$ is indeed the collection of all possible operating points $\mathbf{V}$ that can be found through (3) associated with different values of $\left(X_{1}, X_{2}, \ldots, X_{m}\right)$. It is desirable to find out whether there exists a matrix $\mathbf{M}$ for which the recoverable region $\mathcal{M}_{V}(\mathbf{M})$ covers a large subset of $\mathbb{C}^{n}$, that is relative to the practical solutions of power flow problem. Addressing this problem is central to this paper.

Our first contribution is related to the classical power flow problem. We aim to prove that if the matrix $\mathbf{M}$ satisfies the three properties

- $\mathbf{M} \succeq 0$,

- 0 is a simple eigenvalue of $\mathbf{M}$,

- The all-ones vector $\mathbf{1}_{n}$ belongs to the null space of $\mathbf{M}$, then the region $\mathcal{M}_{V}(\mathbf{M})$ contains the nominal point $(1,1, \ldots, 1)$ and a ball around it. In other words, as long as the specifications $X_{1}, \ldots, X_{2 n-1}$ correspond to a vector of voltages with small angles, the exact recovery of the solution is guaranteed through the proposed SDP problem, without requiring any assumption on the network topology whatsoever. This implies that although the DC model can be used to find an approximate solution around the nominal point, the SDP relaxation would find an exact solution.

It is desirable to find a matrix $\mathbf{M}$ for which the recoverable set $\mathcal{M}_{V}(\mathbf{M})$ is as large as possible with respect to some meaningful measure. This design problem is cumbersome due to the non-convexity of $\mathcal{M}_{V}(\mathbf{M})$. However, we show that the problem of designing a matrix $\mathbf{M}$ for which $\mathcal{M}_{V}(\mathbf{M})$ contains a pre-specified set of voltage vectors $\mathrm{V}$ 's can be cast as a convex program.

Although we develop our results in the context of the classical PF problem, they all hold in the case where $m>$ $2 n-1$, corresponding to redundant specifications. In fact, the proposed approach is flexible in terms of the choice for the types and number of equations, which makes is adoptable for the specification problem. We will demonstrate in simulations that whenever extra equations are available (i.e., $m>2 n-1$ ), the search for a feasible vector of voltages for problem (1) is more likely to be successful via the convex program (3).

\section{Notations}

The symbols $\mathbb{R}$ and $\mathbb{C}$ denote the sets of real and complex numbers, respectively. $\mathbb{S}^{n}$ denotes the space of $n \times n$ real symmetric matrices and $\mathbb{H}^{n}$ denotes the space of $n \times n$ complex Hermitian matrices. $\operatorname{Re}\{\cdot\}, \operatorname{Im}\{\cdot\}, \operatorname{rank}\{\cdot\}, \operatorname{trace}\{\cdot\}$, and $\operatorname{det}\{\cdot\}$ denote the real part, imaginary part, rank, trace, and determinant of a given scalar/matrix. $\operatorname{diag}\{\cdot\}$ denotes the vector of diagonal entries of a matrix. $\|\cdot\|_{F}$ denotes the Frobenius norm of a matrix. Matrices are shown by capital and bold letters. The symbols $(\cdot)^{\mathrm{T}}$ and $(\cdot)^{*}$ denote transpose and conjugate transpose, respectively. Also, "i" is reserved to denote the imaginary unit. The notation $\langle\mathbf{A}, \mathbf{B}\rangle$ represents $\operatorname{trace}\left\{\mathbf{A}^{*} \mathbf{B}\right\}$, which is the inner product of $\mathbf{A}$ and $\mathbf{B}$. The 
notations $\measuredangle x$ and $|x|$ denote the angle and magnitude of a complex number $x$. The notation $\mathbf{W} \succeq 0$ means that $\mathbf{W}$ is a Hermitian and positive semidefinite matrix. Also $\mathbf{W} \succ 0$ means that it is Hermitian and positive definite. The $(i, j)$ entry of $\mathbf{W}$ is denoted as $W_{i j} . \mathbf{0}_{n}$ and $\mathbf{1}_{n}$ denote the $n \times 1$ vectors of zeros and ones, respectively. $\mathbf{0}_{m \times n}$ denotes the $m \times n$ zero matrix and $\mathbf{I}_{n \times n}$ is the $n \times n$ identity matrix. The notation $|\mathcal{X}|$ denotes the cardinality of a set $\mathcal{X}$. For an $m \times n$ matrix $\mathbf{W}$, the notation $\mathbf{W}[\mathcal{X}, \mathcal{Y}]$ denotes the submatrix of $\mathbf{W}$ whose rows and columns are chosen form $\mathcal{X}$ and $\mathcal{Y}$, respectively, for given index sets $\mathcal{X} \subseteq\{1, \ldots, m\}$ and $\mathcal{Y} \subseteq\{1, \ldots, n\}$. Similarly, $\mathbf{W}[\mathcal{X}]$ denotes the submatrix of $\mathbf{W}$ induced by those rows of $\mathbf{W}$ indexed by $\mathcal{X}$. The interior of a set $\mathcal{D} \in \mathbb{C}^{n}$ is denoted as $\operatorname{int}\{\mathcal{D}\}$.

\section{Preliminaries}

Define $\mathbf{P}=\left[\begin{array}{lllll}P_{1} & P_{2} & \cdots & P_{n}\end{array}\right]^{T}$ and $\mathbf{Q}=\left[\begin{array}{llll}Q_{1} & Q_{2} & \cdots & Q_{n}\end{array}\right]^{T}$ as the vectors containing net injected active and reactive powers, respectively. Define also $\mathcal{P}, \mathcal{Q}$ and $\mathcal{V}$ as the sets of buses for which active powers, reactive powers and voltage magnitudes are known, respectively. Let $\mathcal{O}$ denote the set of all buses except the slack bus. The admittance matrix of the network is denoted as $\mathbf{Y}=\mathbf{G}+\mathbf{B i}$, where $\mathbf{G}$ and $\mathbf{B}$ are the conductance and susceptance matrices, respectively. Although the results to be developed in this paper hold for a general matrix $\mathbf{Y}$, we make a few assumptions to streamline the presentation:

- The network is a connected graph.

- Every line of the network consists of a series impedance with nonnegative resistance and inductance.

- The shunt elements are ignored for simplicity in studying the observability of the network, which ensures that

$$
\mathbf{Y} \times \mathbf{1}_{n}=\mathbf{0}_{n}
$$

The following lemma reveals an interesting property of the matrix B, which will later be used in the paper.

Lemma 1. For every $\mathcal{N}^{\prime} \subseteq \mathcal{N}$, we have

$$
\mathbf{B}\left[\mathcal{N}^{\prime}, \mathcal{N}^{\prime}\right] \preceq 0
$$

and $\mathbf{B}\left[\mathcal{N}^{\prime}, \mathcal{N}^{\prime}\right]$ is singular if and only if $\mathcal{N}^{\prime}=\mathcal{N}$.

Proof. B is symmetric and according to (4), we have $\mathbf{B} \mathbf{1}_{n}=$ $\mathbf{0}_{n}$ and since every off-diagonal entry of $\mathbf{B}$ is nonnegative, we have

$$
-B_{k k}=\sum_{k \neq l} B_{k l} \quad \Rightarrow \quad-B_{k k} \geq \sum_{k \neq l}\left|B_{k l}\right|
$$

for every $k \in\{1, \ldots, n\}$. Therefore $-\mathbf{B}$ is diagonally dominant and positive semidefinite and (5) holds for every principal submatrix of $\mathbf{B}$.

Since the network is connected by assumption and every entry of $\mathbf{B}$ corresponding to an existing line of the network is positive, it follows from the Weighted Matrix-Tree Theorem [19] that if $\left|\mathcal{N}^{\prime}\right|=n-1$, then $\operatorname{det}\left\{\mathbf{B}\left[\mathcal{N}^{\prime}, \mathcal{N}^{\prime}\right]\right\} \neq 0$ and consequently $\mathbf{B}\left[\mathcal{N}^{\prime}, \mathcal{N}^{\prime}\right] \prec 0$. In addition, if $\left|\mathcal{N}^{\prime}\right|<n-1$, there exists a set $\mathcal{N}^{\prime \prime} \subset \mathcal{N}$ such that $\mathcal{N}^{\prime} \subset \mathcal{N}^{\prime \prime}$ and $\left|\mathcal{N}^{\prime \prime}\right|=$ $n-1$. According to the Cauchy Interlacing Theorem, every eigenvalue of $\mathbf{B}\left[\mathcal{N}^{\prime}, \mathcal{N}^{\prime}\right]$ is less than or equal to the largest eigenvalue of $\mathbf{B}\left[\mathcal{N}^{\prime \prime}, \mathcal{N}^{\prime \prime}\right]$, which implies that $\mathbf{B}\left[\mathcal{N}^{\prime}, \mathcal{N}^{\prime}\right]$ is non-singular.

Recall that the power balance equations can be expressed as

$$
\mathbf{P}+\mathbf{i} \mathbf{Q}=\operatorname{diag}\left\{\mathbf{V} \mathbf{V}^{*} \mathbf{Y}^{*}\right\} .
$$

Hence, the SDP program (3) associated with the classical PF problem can be written as

$$
\begin{array}{cll}
\underset{\mathbf{W} \in \mathbb{H}^{n}}{\operatorname{minimize}} & \langle\mathbf{W}, \mathbf{M}\rangle & \\
\text { subject to } & \left\langle\mathbf{W}, \mathbf{E}_{k}\right\rangle=\left|V_{k}\right|^{2}, & k \in \mathcal{V} \\
& \left\langle\mathbf{W}, \mathbf{Y}_{Q ; k}\right\rangle=Q_{k}, & k \in \mathcal{Q} \\
& \left\langle\mathbf{W}, \mathbf{Y}_{P ; k}\right\rangle=P_{k}, & \\
& \mathbf{W} \succeq 0 &
\end{array}
$$

where

$$
\begin{aligned}
\mathbf{E}_{k} & \triangleq e_{k} e_{k}^{*} \\
\mathbf{Y}_{Q ; k} & \triangleq \frac{1}{2 \mathbf{i}}\left(\mathbf{Y}_{k}^{*} e_{k} e_{k}^{*}-e_{k} e_{k}^{*} \mathbf{Y}\right) \\
\mathbf{Y}_{P ; k} & \triangleq \frac{1}{2}\left(\mathbf{Y}^{*} e_{k} e_{k}^{*}+e_{k} e_{k}^{*} \mathbf{Y}\right)
\end{aligned}
$$

and $e_{1}, \ldots, e_{n}$ denote the standard basis vectors in $\mathbb{R}^{n}$. The problem (8) is in the canonical form (3) after noticing that

- $\mathbf{M}_{1}, \mathbf{M}_{2}, \ldots, \mathbf{M}_{m}$ correspond to the $m$ matrices $\mathbf{E}_{1}, \mathbf{E}_{2}$, $\ldots, \mathbf{E}_{|\mathcal{V}|}, \quad \mathbf{Y}_{Q ; 1}, \mathbf{Y}_{Q ; 2} \ldots, \mathbf{Y}_{Q ;|\mathcal{Q}|}$ and $\mathbf{Y}_{P ; 1}, \mathbf{Y}_{P ; 2}, \ldots$, $\mathbf{Y}_{P ;|\mathcal{P}|}$.

- The specifications $X_{1}, X_{2}, \ldots, X_{n}$ correspond to $\left|V_{k}\right|^{2}$ 's, $Q_{k}$ 's, and $P_{k}$ 's.

Since the voltage angle at the slack bus is set to zero, the operating point of the power system can be characterized in terms of the real-valued vector

$$
\overline{\mathbf{V}} \triangleq\left[\operatorname{Re}\left\{\mathbf{V}[\mathcal{N}]^{\mathrm{T}}\right\} \quad \operatorname{Im}\left\{\mathbf{V}[\mathcal{O}]^{\mathrm{T}}\right\}\right]^{\mathrm{T}} \in \mathbb{R}^{2 n-1}
$$

Definition 3. Define the function $s(\overline{\mathbf{V}}): \mathbb{R}^{2 n-1} \rightarrow \mathbb{R}^{m}$ as the mapping from the state of the power network $\overline{\mathbf{V}}$ to the vector of specifications $\mathbf{X}$. The $i$-th component of $s(\overline{\mathbf{V}})$ is given by

$$
s_{i}(\overline{\mathbf{V}})=\left\langle\mathbf{V V}^{*}, \mathbf{M}_{i}\right\rangle, \quad i=1, \ldots, m .
$$

Define also the sensitivity matrix $\mathbf{J}_{s}(\overline{\mathbf{V}}) \in \mathbb{R}^{m \times(2 n-1)}$ as the Jacobian of $s(\overline{\mathbf{V}})$ at point $\overline{\mathbf{V}}$, which is equal to

$$
\mathbf{J}_{s}(\overline{\mathbf{V}})=2\left[\begin{array}{lllll}
\overline{\mathbf{M}}_{1} & \overline{\mathbf{V}} & \overline{\mathbf{M}}_{2} \overline{\mathbf{V}} & \ldots & \overline{\mathbf{M}}_{m} \overline{\mathbf{V}}
\end{array}\right]
$$

where

$$
\overline{\mathbf{M}}_{i} \triangleq\left[\begin{array}{cc}
\operatorname{Re}\left\{\mathbf{M}_{i}[\mathcal{N}, \mathcal{N}]\right\} & -\operatorname{Im}\left\{\mathbf{M}_{i}[\mathcal{N}, \mathcal{O}]\right\} \\
\operatorname{Im}\left\{\mathbf{M}_{i}[\mathcal{O}, \mathcal{N}]\right\} & \operatorname{Re}\left\{\mathbf{M}_{i}[\mathcal{O}, \mathcal{O}]\right\}
\end{array}\right]
$$

for every $i=1, \ldots, m$.

\section{MAin Results}

With no loss of generality, we focus on the classical PF problem in this section. Therefore, we assume that $\mathcal{P}$ is the union of PQ and PV buses, $\mathcal{Q}$ is the set of PQ buses, and $\mathcal{V}$ consists of all PV buses as well as the slack bus. Recall that $m$ is equal to $2 n-1$ for the classical PF problem. 


\section{A. Invertibility}

The point $\overline{\mathbf{V}}=\overline{\mathbf{1}}_{n}$ (associated with $\mathbf{V}=\mathbf{1}_{n}$ ) is often regarded as a convenient state for linearization of the power network. According to the inverse function theorem, the invertibility of $\mathbf{J}_{s}\left(\overline{\mathbf{1}}_{n}\right)$ guarantees that the inverse of the function $s(\overline{\mathbf{V}})$ exists in a neighborhood of the point $\overline{\mathbf{1}}_{n}$. Similarly, it follows from Kantorovich Theorem that, under the same invertibility assumption, the power flow problem (1) can be solved using Newton's method by starting from the initial point $\mathbf{1}_{n}$, provided that there exists a solution sufficiently close to this point. The invertibility of $\mathbf{J}_{s}\left(\overline{\mathbf{1}}_{n}\right)$ is beneficial not only for Newton's method but also for the SDP problem. This condition will be explored below.

Lemma 2. The matrix $\mathbf{J}_{s}\left(\overline{\mathbf{1}}_{n}\right)$ is non-singular.

Proof. It is straightforward to verify that

$$
\mathbf{J}_{s}\left(\overline{\mathbf{1}}_{n}\right)=\left[\begin{array}{ccc}
2 \mathbf{I}_{n \times n}[\mathcal{N}, \mathcal{V}] & \mathbf{B}[\mathcal{N}, \mathcal{Q}] & \mathbf{G}[\mathcal{N}, \mathcal{P}] \\
\mathbf{0}_{(n-1) \times m_{V}} & -\mathbf{G}[\mathcal{P}, \mathcal{Q}] & \mathbf{B}[\mathcal{P}, \mathcal{P}]
\end{array}\right] .
$$

By Gaussian elimination, $\mathbf{J}_{s}\left(\overline{\mathbf{1}}_{n}\right)$ reduces to the matrix

$$
\mathbf{S} \triangleq\left[\begin{array}{cc}
\mathbf{B}[\mathcal{Q}, \mathcal{Q}] & \mathbf{G}[\mathcal{Q}, \mathcal{P}] \\
-\mathbf{G}[\mathcal{P}, \mathcal{Q}] & \mathbf{B}[\mathcal{P}, \mathcal{P}]
\end{array}\right]
$$

Hence, it suffices to prove that $\mathbf{S}$ is not singular. To this end, one can write

$$
\operatorname{det}\{\mathbf{S}\}=\operatorname{det}\left\{\mathbf{S}_{1}\right\} \operatorname{det}\left\{\mathbf{S}_{2}\right\},
$$

where $\mathbf{S}_{1} \triangleq \mathbf{B}[\mathcal{P}, \mathcal{P}]$ and $\mathbf{S}_{2}$ is the Schur complement of $\mathbf{S}_{1}$ in $\mathbf{S}$, i.e.,

$$
\mathbf{S}_{2} \triangleq \mathbf{B}[\mathcal{Q}, \mathcal{Q}]+\mathbf{G}[\mathcal{Q}, \mathcal{P}] \mathbf{B}[\mathcal{P}, \mathcal{P}]^{-1} \mathbf{G}[\mathcal{P}, \mathcal{Q}] .
$$

On the other hand, $\mathbf{S}_{1}$ and $\mathbf{S}_{2}$ are both symmetric, and in addition Lemma 1 yields that $\mathbf{S}_{1} \prec 0$ and $\mathbf{B}[\mathcal{Q}, \mathcal{Q}] \prec 0$. This implies that $\mathbf{S}_{2} \prec 0$ according to the above equation, which leads to the relation $\operatorname{det}\{\mathbf{S}\} \neq 0$.

Remark 1. It is straightforward to verify that Lemma 2 is true for arbitrary networks with shunt elements as long as the matrix $\mathbf{Y}$ is generic. In other words, if $\mathbf{J}_{s}\left(\overline{\mathbf{1}}_{n}\right)$ is singular for an arbitrary power network, an infinitesimal perturbation of the susceptance values of the existing lines makes the resulting $\mathbf{J}_{s}\left(\overline{\mathbf{1}}_{n}\right)$ non-singular.

Definition 4. Define $\mathcal{J}$ as the set of all voltage vectors $\mathbf{V}$ for which $\mathbf{J}_{s}\left(\overline{\mathbf{V}}_{n}\right)$ is non-singular.

\section{B. Region of Recoverable Voltages}

Given a matrix $\mathbf{M}$, we intend to characterize $\mathcal{M}_{V}(\mathbf{M})$, i.e., the set of all voltage vectors that can be recovered using the convex problem (3). In what follows, we first explain the main results of this work and then prove them in Subsection III-C.

Definition 5. For every vector $\mathbf{V} \in \mathcal{J}$, define

$$
\Lambda(\mathbf{V}, \mathbf{M}) \triangleq-2 \mathbf{J}_{s}(\overline{\mathbf{V}})^{-1} \overline{\mathbf{M}} \overline{\mathbf{V}}
$$

where

$$
\overline{\mathbf{M}}=\left[\begin{array}{cc}
\operatorname{Re}\{\mathbf{M}[\mathcal{N}, \mathcal{N}]\} & -\operatorname{Im}\{\mathbf{M}[\mathcal{N}, \mathcal{O}]\} \\
\operatorname{Im}\{\mathbf{M}[\mathcal{O}, \mathcal{N}]\} & \operatorname{Re}\{\mathbf{M}[\mathcal{O}, \mathcal{O}]\}
\end{array}\right]
$$

Define also

$$
f(\mathbf{V}, \mathbf{M}) \triangleq \mathbf{M}+\sum_{i=1}^{2 n-1} \Lambda_{i}(\mathbf{V}, \mathbf{M}) \mathbf{M}_{i}
$$

where $\Lambda_{i}(\mathbf{V}, \mathbf{M})$ denotes the $i^{\text {th }}$ entry of $\Lambda(\mathbf{V}, \mathbf{M})$.

Definition 6. For every $\varepsilon \geq 0$, define $\mathcal{D}_{\varepsilon}$ as the set of all positive semidefinite Hermitian matrices whose sum of two smallest eigenvalues is greater than $\varepsilon$. Also, denote $\mathcal{D}_{0}$ as $\mathcal{D}$.

Since the sum of the two smallest eigenvalues of a Hermitian matrix variable is a concave function of that matrix, the set $\mathcal{D}$ is convex. The first result of this work will be provided below.

Theorem 1. The interior of the set $\mathcal{M}_{V}(\mathbf{M})$ can be characterized as

$$
\operatorname{int}\left\{\mathcal{M}_{V}(\mathbf{M})\right\} \cap \mathcal{J}=\{\mathbf{V} \in \mathcal{J} \mid f(\mathbf{V}, \mathbf{M}) \in \mathcal{D}\} .
$$

The above theorem offers a nonlinear matrix inequality to characterize the interior of the set of recoverable voltage vectors, except for a subset of measure zero of this interior at which the Jacobian of $s(\overline{\mathbf{V}})$ loses rank. A question arises as to whether this interior is non-empty. This problem will be addressed next.

Assumption 1. The matrix $\mathbf{M}$ satisfies the following properties:

- $\mathbf{M} \succeq 0$

- 0 is a simple eigenvalue of $\mathbf{M}$

- The vector $\mathbf{1}_{n}$ belongs to the null space of $\mathbf{M}$.

Theorem 2. Consider an arbitrary matrix $M$ satisfying the Assumption 1. The region $\mathcal{M}_{V}(\mathbf{M})$ has a non-empty interior containing the point $\mathbf{1}_{n}$.

Due to (4), if $\mathbf{M}$ is chosen as $\mathbf{Y}^{*} \mathbf{Y}$, it will satisfy the Assumption 1. In that case, the objective of the convex problem (3) corresponds to $\left|I_{1}\right|^{2}+\left|I_{2}\right|^{2}+\cdots+\left|I_{n}\right|^{2}$, where $I_{k}$ denotes the current at bus $k$. In that case, Theorem 2 states that as long as the voltage angles are small enough, a solution of the PF problem can be recovered exactly by means of an SDP relaxation whose objective function reflects the minimization of nodal currents.

There are infinitely many M's satisfying the Assumption 1 , each resulting in a potentially different recoverable set $\mathcal{M}_{V}(\mathbf{M})$. To find the best $M$, one can search for a set $\mathcal{M}_{V}(\mathbf{M})$ with the maximum volume or containing the largest ball. However, these problems are indeed hard to solve due to the non-convexity of $\mathcal{M}_{V}(\mathbf{M})$. Another approach for seeking a good $\mathbf{M}$ is to first choose a set of voltage vectors scattered in $\mathbb{C}^{n}$ and then find a matrix $\mathbf{M}$ (if any) whose recoverable set $\mathcal{M}_{V}(\mathbf{M})$ contains all these points. It turns out that this design problem is in fact convex.

Theorem 3. Given $r$ arbitrary points $\widehat{\mathbf{V}}_{1}, \widehat{\mathbf{V}}_{2}, \ldots, \widehat{\mathbf{V}}_{r} \in \mathcal{J}$, 
consider the problem

find $\quad \mathbf{M} \in \mathbb{H}^{n}$

subject to $\quad f\left(\widehat{\mathbf{V}}_{l}, \mathbf{M}\right) \in \mathcal{D}_{\varepsilon}, \quad l=1,2, \ldots, r$

$$
\mathbf{M} \in \mathcal{D}_{\varepsilon}
$$$$
\mathbf{M} \times \mathbf{1}_{n}=\mathbf{0}_{n}
$$

where $\varepsilon>0$ is an arbitrary constant. The following statements hold:

i) The feasibility problem (11) is convex.

ii) There exists a matrix $\mathbf{M}$ satisfying the Assumption 1 such that the associated recoverable set $\mathcal{M}_{V}(\mathbf{M})$ contains $\widehat{\mathbf{V}}_{1}, \widehat{\mathbf{V}}_{2}, \ldots, \widehat{\mathbf{V}}_{r}$ and a ball around each of these points if and only if the convex problem (11) has a solution $\mathbf{M}$.

\section{Proofs}

In this part, we will prove Theorems 1,2 and 3 . To this end, it is useful to derive the dual of (3). This problem can be stated as

$$
\begin{array}{ll}
\underset{\mathbf{L} \in \mathbb{R}^{2 n-1}}{\operatorname{minimize}} & \mathbf{X}^{\mathrm{T}} \mathbf{L} \\
\text { subject to } & \mathbf{M}+\sum_{i=1}^{2 n-1} L_{i} \mathbf{M}_{i} \succeq 0
\end{array}
$$

where the dual variable $\mathbf{L}$ is the vector of all Lagrange multipliers $L_{1}, \ldots, L_{2 n-1}$, and $\mathbf{X}=\left[\begin{array}{llll}X_{1} & X_{2} & \cdots & X_{m}\end{array}\right]^{T}$. For every $L \in \mathbb{R}^{2 n-1}$, define

$$
\mathbf{A}(\mathbf{L})=\mathbf{M}+\sum_{i=1}^{2 n-1} L_{i} \mathbf{M}_{i}
$$

We need to develop three lemmas before proving Theorem 1 .

Lemma 3. Consider two arbitrary vectors $\mathbf{V} \in \mathcal{J}$ and $\mathbf{L} \in$ $\mathbb{R}^{2 n-1}$. The relation

$$
\mathbf{A}(\mathbf{L}) \mathbf{V}=0
$$

holds if and only if $\mathbf{L}=\Lambda(\mathbf{V}, \mathbf{M})$.

Proof. Equation (14) can be rearranged as

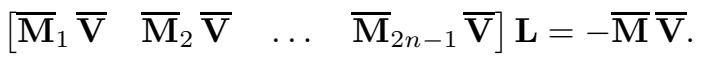

Hence, due to the invertiblity of $\mathbf{J}_{s}(\overline{\mathbf{V}})$ and Definition 5, $\Lambda(\mathbf{V}, \mathbf{M})$ is the unique solution of (15).

The following Lemma studies the recoverability of a voltage vector $\mathbf{V}$ via the convex problem (3).

Lemma 4. Assume that $\mathbf{V}$ is a feasible solution of the power flow problem (1) such that $\mathbf{J}_{s}(\overline{\mathbf{V}})$ is invertible. Then, strong duality holds between the primal problem (3) and the dual problem (12). In addition, the following two statements are equivalent:

i) $\mathbf{V V}^{*}$ is an optimal solution for the primal problem (3).

ii) $\Lambda(\mathbf{V}, \mathbf{M})$ is a feasible point for the dual problem (12).

Proof. By assumption, the matrix $\mathbf{V V}^{*}$ is a feasible point for the problem (3). In order to show the strong duality, it suffices to build a strictly feasible point $\tilde{\mathbf{L}}$ for the dual problem. To this end, we set the Lagrange multipliers corresponding to active power, reactive power and voltage magnitude specifications equal to $0,-1$, and a constant $c$, respectively. Then, one can write

$$
\begin{aligned}
\mathbf{A}(\tilde{\mathbf{L}})[\mathcal{Q}, \mathcal{Q}] & =-\mathbf{B}[\mathcal{Q}, \mathcal{Q}] \\
\mathbf{A}(\tilde{\mathbf{L}})[\mathcal{V}, \mathcal{V}] & =c \mathbf{I}_{|\mathcal{V}| \times|\mathcal{V}|}
\end{aligned}
$$

(recall that $\mathbf{A}(\tilde{\mathbf{L}})[\mathcal{Q}, \mathcal{Q}]$ denotes a submatrix of $\mathbf{A}(\tilde{\mathbf{L}})$ induced by the index set $\mathcal{Q}$ ). Since $\mathcal{V} \cup \mathcal{Q}=\mathcal{N}$ and $\mathcal{V}$ has at least one member (the slack bus), $\mathbf{B}[\mathcal{Q}, \mathcal{Q}]$ is negative definite according to Lemma 1. Now, the strict positive definiteness of $\mathbf{A}(\tilde{\mathbf{L}})$ can be ensured by choosing a sufficiently large $c$.

$(i) \Rightarrow(i i)$ : Due to strong duality, if $\mathbf{V} \mathbf{V}^{*}$ is primal optimal, there exists a vector $\mathbf{L}^{\prime} \in \mathbb{R}^{2 n-1}$ that is dual feasible:

$$
\mathbf{A}\left(\mathbf{L}^{\prime}\right) \succeq 0
$$

and satisfies the complementary slackness:

$$
\left\langle\mathbf{V} \mathbf{V}^{*}, \mathbf{A}\left(\mathbf{L}^{\prime}\right)\right\rangle=0
$$

Hence,

$$
\left\langle\mathbf{V} \mathbf{V}^{*}, \mathbf{A}\left(\mathbf{L}^{\prime}\right)\right\rangle=\operatorname{trace}\left\{\mathbf{V} \mathbf{V}^{*} \mathbf{A}\left(\mathbf{L}^{\prime}\right)\right\}=\mathbf{V}^{*} \mathbf{A}\left(\mathbf{L}^{\prime}\right) \mathbf{V}=0 .
$$

It follows from this equation and (18) that $\mathbf{A}\left(\mathbf{L}^{\prime}\right) \mathbf{V}=0$. The, according to Lemma 3 , we have $\mathbf{L}^{\prime}=\Lambda(\mathbf{V}, \mathbf{M})$, which implies that $\Lambda(\mathbf{V}, \mathbf{M})$ is dual feasible.

(ii) $\Rightarrow(i)$ : It is shown in Lemma 3 that $\mathbf{A}(\Lambda(\mathbf{V}, \mathbf{M})) \mathbf{V}=$ 0 . Therefore,

$$
\left\langle\mathbf{V} \mathbf{V}^{*}, \mathbf{A}(\Lambda(\mathbf{V}, \mathbf{M}))\right\rangle=0 .
$$

As a result, $\Lambda(\mathbf{V}, \mathbf{M})$ acts as a dual certificate for the optimality of $\mathbf{V V}^{*}$.

Lemma 5. Suppose that zero and $\delta>0$ are the two smallest eigenvalues of an $m \times m$ matrix $\mathbf{A}_{1} \in \mathcal{D}$. Then, for every matrix $\mathbf{A}_{2} \in \mathbb{H}^{m}$ with 0 as its eigenvalue, the relation $\mathbf{A}_{2} \in \mathcal{D}$ holds if $\left\|\mathbf{A}_{1}-\mathbf{A}_{2}\right\|_{F}<\sqrt{\delta}$.

Proof. Let $\mathbf{Q} \Delta \mathbf{Q}^{*}$ denote the eigenvalue decomposition of $\mathbf{A}_{1}$ such that $\Delta_{m m}=0$. One can decompose $\Delta$ and $\mathbf{A}_{2}$ as

$$
\Delta=\left[\begin{array}{cc}
\Delta_{1} & \mathbf{0} \\
\mathbf{0} & 0
\end{array}\right] \quad \text { and } \quad \mathbf{Q}^{*} \mathbf{A}_{2} \mathbf{Q}=\left[\begin{array}{cc}
\Delta_{2} & \mathbf{u} \\
\mathbf{u}^{*} & u
\end{array}\right]
$$

for some matrices $\Delta_{1}, \Delta_{2} \in \mathbb{H}^{m-1}, \mathbf{u} \in \mathbb{C}^{m-1}$ and $u \in \mathbb{R}$. Then, for every vector $\mathbf{x} \in \mathbb{C}^{m-1}$ of length 1 , one can write

$$
\begin{aligned}
\mathbf{x}^{*} \Delta_{2} \mathbf{x} & =\mathbf{x}^{*} \Delta_{1} \mathbf{x}-\mathbf{x}^{*}\left(\Delta_{1}-\Delta_{2}\right) \mathbf{x} \\
& \geq \delta-\left\|\Delta_{1}-\Delta_{2}\right\|_{F}^{2} \\
& \geq \delta-\left\|\mathbf{A}_{1}-\mathbf{A}_{2}\right\|_{F}^{2}>0
\end{aligned}
$$

It can be concluded from the above equation that $\Delta_{2} \succ 0$. On the other hand, according to Schur complement, we have

$$
0=\operatorname{det}\left\{\mathbf{A}_{2}\right\}=\operatorname{det}\left\{\Delta_{2}\right\} \times \operatorname{det}\left\{u-\mathbf{u}^{*} \Delta_{2}^{-1} \mathbf{u}\right\},
$$

which implies that $u-\mathbf{u}^{*} \Delta_{2}^{-1} \mathbf{u}=0$. Using Schur complement once more, it can be concluded that $\mathbf{A}_{2} \succeq 0$. Moreover,

$$
\operatorname{rank}\left\{\mathbf{A}_{2}\right\}=\operatorname{rank}\left\{\Delta_{2}\right\}+\operatorname{rank}\left\{u-\mathbf{u}^{*} \Delta_{2}^{-1} \mathbf{u}\right\}=n-1,
$$


implying that $\mathbf{A}_{2} \in \mathcal{D}$.

Proof of Theorem 1: We first need to show that $\{\mathbf{V} \in$ $\mathcal{J} \mid f(\mathbf{V}, \mathbf{M}) \in \mathcal{D}\}$ is an open set. Consider a vector $\mathbf{V}$ such that $f(\mathbf{V}, \mathbf{M}) \in \mathcal{D}$ and let $\delta$ denote the second smallest eigenvalue of $f(\mathbf{V}, \mathbf{M})$. Due to the continuity of $\operatorname{det}\left\{\mathbf{J}_{s}(\cdot)\right\}$ and $f(\cdot, \mathbf{M})$, there exists a neighborhood $\mathcal{B} \in \mathbb{C}^{n}$ around $\mathbf{V}$ such that for every $\mathbf{V}^{\prime}$ within this neighborhood, $f\left(\mathbf{V}^{\prime}, \mathbf{M}\right)$ is well defined (i.e., $\mathbf{V}^{\prime} \in \mathcal{J}$ ) and

$$
\left\|f\left(\mathbf{V}^{\prime}, \mathbf{M}\right)-f(\mathbf{V}, \mathbf{M})\right\|_{F}<\sqrt{\delta} .
$$

Hence, according to Lemma 5, we have $f\left(\mathbf{V}^{\prime}, \mathbf{M}\right) \in \mathcal{D}$ for every $\mathbf{V}^{\prime} \in \mathcal{B}$. This proves that $\{\mathbf{V} \in \mathcal{J} \mid f(\mathbf{V}, \mathbf{M}) \in \mathcal{D}\}$ is an open set.

Now, consider a vector $\mathbf{V} \in \mathcal{J}$ such that $f(\mathbf{V}, \mathbf{M}) \in \mathcal{D}$. The objective is to show that $\mathbf{V} \in \operatorname{int}\left\{\mathcal{M}_{V}(\mathbf{M})\right\}$. Notice that since $f(\mathbf{V}, \mathbf{M})$ is assumed to be in the set $\mathcal{D}$, the vector $\Lambda(\mathbf{V}, \mathbf{M})$ is a feasible point for the dual problem (12). Therefore, it follows from Lemma 4 that the matrix $\mathbf{V V}^{*}$ is an optimal solution for the primal problem (3). In addition, every solution $\mathbf{W}$ must satisfy

$$
\langle f(\mathbf{V}, \mathbf{M}), \mathbf{W}\rangle=0 .
$$

According to Lemma $3, \mathbf{V}$ is an eigenvector of $f(\mathbf{V}, \mathbf{M})$ corresponding to the eigenvalue 0 . Therefore, since $f(\mathbf{V}, \mathbf{M}) \succeq 0$ and $\operatorname{rank}\{f(\mathbf{V}, \mathbf{M})\}=n-1$, every positive semidefinite matrix $\mathbf{W}$ satisfying (26) is equal to $c \mathbf{V} \mathbf{V}^{*}$ for a nonnegative constant $c$. This concludes that $\mathbf{V} \mathbf{V}^{*}$ is the unique solution to (3), and therefore $\mathbf{V}$ belongs to $\mathcal{M}_{V}(\mathbf{M})$. Since $\{\mathbf{V} \in$ $\mathcal{J} \mid f(\mathbf{V}, \mathbf{M}) \in \mathcal{D}\}$ is shown to be an open set, the above result can be translated as

$$
\{\mathbf{V} \in \mathcal{J} \mid f(\mathbf{V}, \mathbf{M}) \in \mathcal{D}\} \subseteq \operatorname{int}\left\{\mathcal{M}_{V}(\mathbf{M})\right\} \cap \mathcal{J}
$$

In order to complete the proof, it is requited to show that $\operatorname{int}\left\{\mathcal{M}_{V}(\mathbf{M})\right\} \cap \mathcal{J}$ is a subset of $\{\mathbf{V} \in \mathcal{J} \mid f(\mathbf{V}, \mathbf{M}) \in \mathcal{D}\}$. To this end, consider a vector $\mathbf{V} \in \operatorname{int}\left\{\mathcal{M}_{V}(\mathbf{M})\right\} \cap \mathcal{J}$. This means that $\mathbf{V} \mathbf{V}^{*}$ is a solution to (3), and therefore $f(\mathbf{V}, \mathbf{M}) \succeq$ 0 , due to Lemma 4 . To prove the aforementioned inclusion by contradiction, suppose that $f(\mathbf{V}, \mathbf{M}) \notin \mathcal{D}$, implying that 0 is an eigenvalue of $f(\mathbf{V}, \mathbf{M})$ with multiplicity at least 2 . Let $\mathbf{U}$ denote a second eigenvector corresponding to the eigenvalue 0 such that $\mathbf{V}^{*} \mathbf{U}=0$. Since $\mathbf{J}_{s}(\overline{\mathbf{V}}) \neq 0$, in light of the inverse function theorem, there exists a constant $\varepsilon_{0}>0$ with the property that for every $\varepsilon \in\left[0, \varepsilon_{0}\right]$, there is a vector $\mathbf{T}_{\varepsilon} \in \mathbb{C}^{n}$ satisfying the relation

$$
s\left(\overline{\mathbf{T}}_{\varepsilon}\right)=s(\overline{\mathbf{V}})+\varepsilon s(\overline{\mathbf{U}})
$$

where the function $s(\cdot)$ is defined in Definition 3. This means that the rank-2 matrix

$$
\mathbf{W}=\mathbf{V} \mathbf{V}^{*}+\varepsilon \mathbf{U}^{*}
$$

is a solution to the problem (3) associated with the dual certificate $\Lambda(\mathbf{V}, \mathbf{M})$, and therefore $\mathbf{T}_{\varepsilon} \notin \mathcal{M}_{V}(\mathbf{M})$. This contradicts the previous assumption that $\mathbf{V} \in \operatorname{int}\left\{\mathcal{M}_{V}(\mathbf{M})\right\}$. Therefore, we have $f(\mathbf{V}, \mathbf{M}) \in \mathcal{D}$, which completes the proof.
Proof of Theorem 2: It can be inferred from Lemma 2 that $\overline{\mathbf{1}}_{n} \in \mathcal{J}$. On the other hand, since $\mathbf{M} \times \mathbf{1}_{n}=0$, we have

$$
\Lambda\left(\mathbf{1}_{n}, \mathbf{M}\right)=\mathbf{0}_{2 n-1},
$$

which concludes that

$$
f\left(\mathbf{1}_{n}, \mathbf{M}\right)=\mathbf{M} \in \mathcal{D} .
$$

Therefore, it follows from Theorem 1 that $\mathbf{1}_{n} \in$ $\operatorname{int}\left\{\mathcal{M}_{V}(\mathbf{M})\right\}$.

Proof of Theorem 3: Part (i) is proved by noting that the sum of the two smallest eigenvalues of a matrix is a concave function and that $f\left(\widehat{\mathbf{V}}_{l}, \mathbf{M}\right)$ is a linear function with respect to $\mathrm{M}$.

Part (ii) follows immediately from Theorems 1 and 2.

\section{ILLUSTRATIVE EXAMPLES}

Example 1: Consider a 3-bus power network with the line admittances

$$
\begin{aligned}
& y_{12}=-2 \mathbf{i}, \\
& y_{13}=-1 \mathbf{i}, \\
& y_{23}=-3 \mathbf{i} .
\end{aligned}
$$

Assume that the voltage magnitudes are all equal to 1 , and that the active powers $P_{1}, P_{2}, P_{3}$ are all given. The PF problem aims to find the complex voltage vector $\mathbf{V}$. To this end, let $\mathbf{V}$ be parameterized as

$$
\mathbf{V}=\left[\begin{array}{lll}
1 & e^{-\theta_{2} \mathbf{i}} & e^{-\theta_{3} \mathbf{i}}
\end{array}\right]^{*}
$$

where $\theta_{2}, \theta_{3} \in\left[-180^{\circ}, 180^{\circ}\right]$. Consider the convex problem (3) with $M$ equal to $\mathbf{Y}^{*} \mathbf{Y}$. This optimization solves the $\mathrm{PF}$ problem exactly if and only if $\left(\theta_{2}, \theta_{3}\right)$ belongs to the region depicted in Figure 1(a). Alternatively, the above convex optimization finds a solution of the PF problem if and only if $\left(P_{1}, P_{2}\right)$ is contained in the region provided in Figure 1(b). On the other hand, the set of all feasible vectors $\left(P_{1}, P_{2}\right)$ for the power network specified by (32) is drawn in Figure 1(c). By comparing Figures 1(b) and 1(c), it can be concluded that the convex optimization (3) finds a solution of $\mathrm{PF}$ for every feasible vector $\left(P_{1}, P_{2}, P_{3}\right)$ (note that $P_{3}=-P_{1}-P_{2}$ ). However, the reason why the angle region in Figure 1(a) is not the entire box $[-180,180] \times[-180,180]$ is that some instances of the PF problem have multiple solutions (multiple values for the pair $\left.\left(\theta_{2}, \theta_{3}\right)\right)$ and the proposed convex optimization finds just one of those solutions.

Example 2: This example is similar to Example 1 with the only difference that $y_{12}$ is changed to $2 \mathbf{i}$. The outcomes are plotted in Figure 2. It can be seen in Fig 2(a) that the set of values for $\left(\theta_{2}, \theta_{3}\right)$ that can be successfully recovered by the convex optimization (3) is connected but non-convex. Although this non-convexity is observed here for a nonrealistic (negative) line impedance in a 3-bus network, the same phenomenon occurs for larger networks with legitimate positive inductances.

Example 3: This example is similar to Example 1 with the only difference that $y_{23}$ is changed to $4 \mathbf{i}$. The outcomes are plotted in Figure 3. It can be seen in Fig 3(a) that the set of 


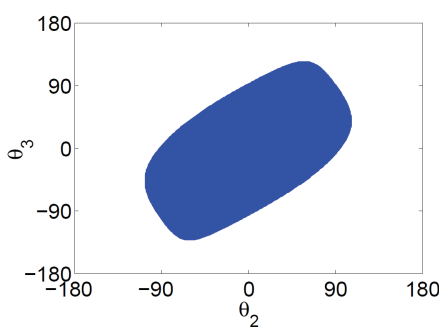

(a)

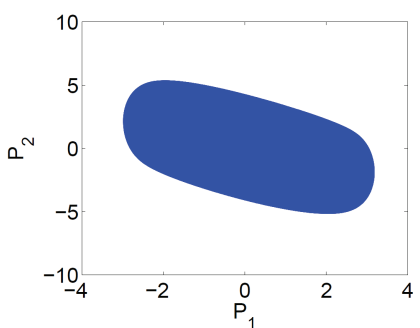

(b)

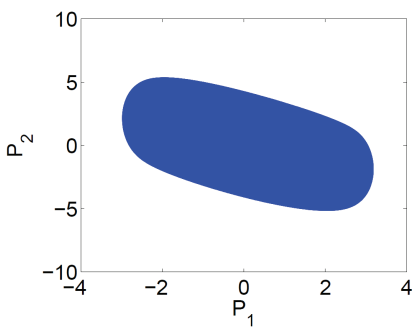

(c)

Fig. 1: These plots show the outcome of the convex problem (3) for Example 1. (a): the angle region that can be recovered via problem (3), (b): the power region that can be recovered via problem (3), (c): the entire feasible power region for the 3-bus system.

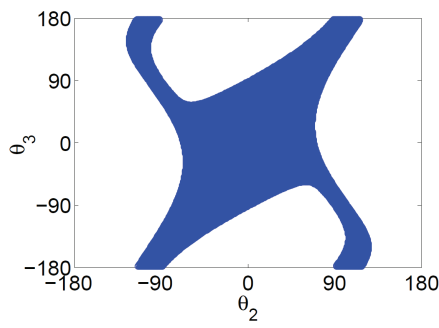

(a)

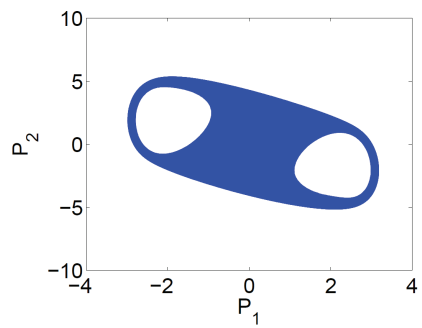

(b)

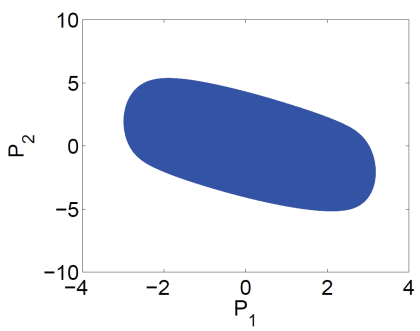

(c)

Fig. 2: These plots show the outcome of the convex problem (3) for Example 2. (a): the angle region that can be recovered via problem (3), (b): the power region for which problem (3), (c): the entire power region for the 3-bus system.

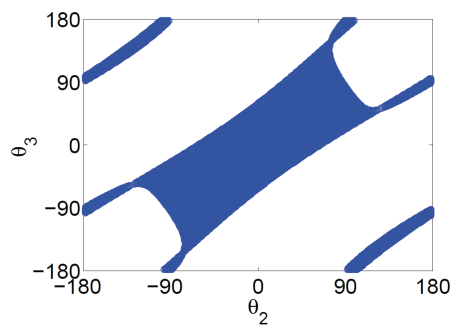

(a)

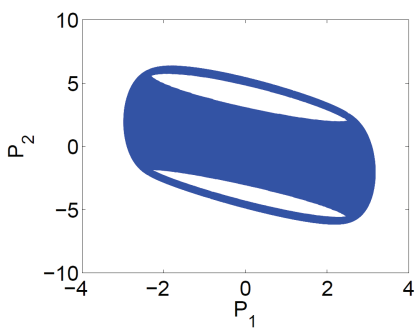

(b)

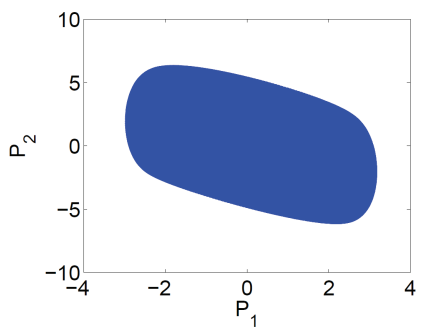

(c)

Fig. 3: These plots show the outcome of the convex problem (3) for Example 3. (a): the angle region that can be recovered via problem (3), (b): the power region that can be recovered via problem (3), (c): the entire feasible power region for the 3-bus system.

values for $\left(\theta_{2}, \theta_{3}\right)$ that can be successfully recovered by the convex optimization (3) is disconnected.

\section{Simulation Results}

In order to evaluate the performance of the SDP relaxation for the PF problem, we perform numerical simulations on the IEEE 9-bus, New England 39-bus, and IEEE 57-bus systems [20]. Three recovery methods are considered for each test case:

1) Newton's method: We evaluate the probability of convergence for Newton's method in polar coordinates for the classical PF problem with $2 n-1$ specifications, where the starting point is $V_{k}=1 \angle 0^{\circ}$ for every $k \in \mathcal{N}$.

2) SDP relaxation: The probability of obtaining a rank-1 solution for the SDP relaxation (3) with $\mathbf{M}=\mathbf{Y}^{*} \mathbf{Y}$ is evaluated, where the same set of specifications as in Newton's method is used.

3) SDP relaxation with extra specifications: The probability of obtaining a rank-1 solution for the SDP relaxation (3) with $\mathbf{M}=\mathbf{Y}^{*} \mathbf{Y}$ is evaluated, under extra specifications compared to the classical PF problem. It is assumed that active powers are measured at $\mathrm{PV}$ and PQ buses, reactive powers are measured at PQ buses, and voltages magnitudes are measured at all buses (as opposed to only PV and slack buses).

For different values of $\theta$, we generated 500 specification sets $\left(X_{1}, \ldots, X_{m}\right)$ by randomly choosing voltage vectors whose magnitudes and phases are uniformly drawn from the intervals $[0.9,1.1]$ and $[-\theta, \theta]$, respectively. We then exploited each 


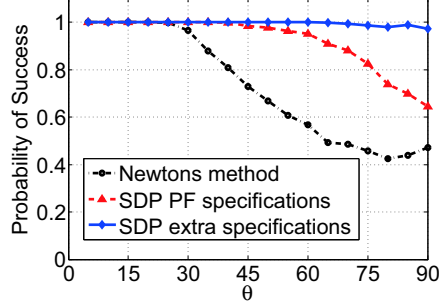

(a)

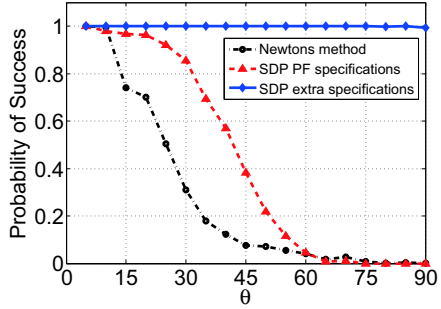

(b)

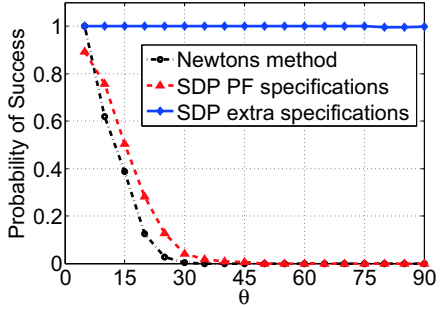

(c)

Fig. 4: These plots show the probability of success for Newton's method, SDP relaxation, and SDP relaxation with extra specifications for (a): IEEE 9-bus system, (b): New England 39-bus system, and (c): IEEE 57-bus system.

of the three above methods to find a feasible voltage vector associated with each specification set. The results are depicted in Figure 4.

\section{CONCLUSION}

In this paper, the classical power flow (PF) problem is studied by means of a semidefinite programming (SDP) relaxation. The proposed method is based on lifting the nonlinear equations to a higher dimension, where the equations can be cast linearly in terms of a positive semidefinite and rank-one matrix variable. This leads to a family of convex optimization problems, each in the form of a semidefinite program with a linear objective function that captures the rank-one constraint as a proxy. The proposed convex optimization problems are guaranteed to solve the PF problem if the voltage angles are small. The region of complex voltages that can be recovered through each problem is characterized by a nonlinear matrix inequality. Moreover, the problem of finding a convenient objective function for SDP that can recover a given set of voltage vectors and a neighborhood around each vector can itself be cast as a convex problem. The simulation results show the superiority of the proposed method over the traditional Newton's method.

\section{REFERENCES}

[1] K. Lehmann, A. Grastien, and P. Van Hentenryck, "AC-feasibility on tree networks is NP-Hard," submitted for publication. [Online]. Available: http://arxiv.org/abs/1410.8253.

[2] A. Verma, "Power grid security analysis: An optimization approach," $\mathrm{Ph} . \mathrm{D}$. dissertation, Columbia University, 2009.

[3] E. J. Candes, T. Strohmer, and V. Voroninski, "Phaselift: Exact and stable signal recovery from magnitude measurements via convex programming," Commun. Pur. Appl. Math., vol. 66, no. 8, pp. 1241-1274, Aug. 2013.

[4] B. Recht, M. Fazel, and P. A. Parrilo, "Guaranteed minimum-rank solutions of linear matrix equations via nuclear norm minimization," SIAM Rev., vol. 52, no. 3, pp. 471-501, Aug. 2010.

[5] R. Madani, G. Fazelnia, S. Sojoudi, and J. Lavaei, "Low-rank solutions of matrix inequalities with applications to polynomial optimization and matrix completion problems," in Conference on Decision and Control, 2014.

[6] J. Lavaei and S. Low, "Zero duality gap in optimal power flow problem," IEEE Trans. Power Syst., vol. 27, no. 1, pp. 92-107, Feb. 2012.

[7] J. Lavaei, "Zero duality gap for classical OPF problem convexifies fundamental nonlinear power problems," in Proc. Amer. Control Conf., June 2011, pp. 4566-4573.

[8] S. Sojoudi and J. Lavaei, "Physics of power networks makes hard optimization problems easy to solve," IEEE Power and Energy Society General Meeting, 2012.
[9] A. Lam, B. Zhang, and D. Tse, "Distributed algorithms for optimal power flow problem," in Proc. IEEE Conf. Decision and Control, 2012.

[10] B. Zhang, A. Lam, A. Dominguez-Garcia, and D. Tse, "An optimal and distributed method for voltage regulation in power distribution systems," IEEE Trans. Power App. Syst., to be published. [Online]. Available: http://arxiv.org/abs/1204.5226.

[11] D. K. Molzahn, J. T. Holzer, B. C. Lesieutre, and C. L. DeMarco, "Implementation of a large-scale optimal power flow solver based on semidefinite programming," IEEE Trans. Power Syst., vol. 28, no. 4, pp. 3987-3998, Nov. 2013

[12] M. S. Andersen, A. Hansson, and L. Vandenberghe, "Reducedcomplexity semidefinite relaxations of optimal power flow problems," IEEE Trans. Power Syst., vol. 29, no. 4, pp. 1855-1863, Jul 2014.

[13] R. Jabr, "Exploiting sparsity in SDP relaxations of the OPF problem," IEEE Trans. Power Syst., vol. 27, no. 2, pp. 1138-1139, May. 2012.

[14] R. Madani, M. Ashraphijuo, and J. Lavaei, "Promises of conic relaxation for contingency-constrained optimal power flow problem," in Proc. Allerton Conf., 2014.

[15] R. Madani, S. Sojoudi, and J. Lavaei, "Convex relaxation for optimal power flow problem: Mesh networks," IEEE Trans. Power Syst., vol. 30, no. 1, pp. 199-211, Jan. 2015.

[16] W. F. Tinney and C. E. Hart, "Power flow solution by Newton's method," IEEE T. Power Ap. Syst., vol. 86, no. Nov., pp. 1449-1460, Jun. 1967.

[17] B. Stott and O. Alsaç, "Fast decoupled load flow," IEEE T. Power Ap. Syst., vol. 93, no. 3, pp. 859-869, May. 1974.

[18] R. A. Van Amerongen, "A general-purpose version of the fast decoupled load flow," IEEE Trans. Power Syst., vol. 4, no. 2, pp. 760-770, May. 1989.

[19] A. Duval, C. Klivans, and J. Martin, "Simplicial matrix-tree theorems," T. Am. Math. Soc., vol. 361, no. 11, pp. 6073-6114, Jun. 2009.

[20] R. D. Zimmerman, C. E. Murillo-Sánchez, and R. J. Thomas, "Matpower's extensible optimal power flow architecture," in Power \& Energy Society General Meeting, 2009. PES'09. IEEE. IEEE, 2009, pp. 1-7. 